\title{
Interessenskonflikte in Leitlinien - wo stehen wir, wo wollen wir hin?
}

\section{Guidelines and Conflicts of Interest: where are we, where do we go from here?}

Autoren

Institute
S. Ewig' ${ }^{1}$ T. Schaberg ${ }^{2}$, T. Welte ${ }^{3}$, J. H. Ficker ${ }^{4}$, M. Pfeifer ${ }^{5}$, B. Schönhofer ${ }^{6}$

Die Institutsangaben sind am Ende des Beitrags gelistet.
Bibliografie

DOI http://dx.doi.org/

$10.1055 / \mathrm{s}-0032-1326062$

Pneumologie 2013; 67: 13-15

(c) Georg Thieme Verlag KG

Stuttgart · New York

ISSN 0934-8387

\section{Korrespondenzadresse}

Prof. Dr. med. Santiago Ewig

Thoraxzentrum Ruhrgebiet

Kliniken für Pneumologie und Infektiologie

Ev. Krankenhaus Herne und

Augusta-Krankenanstalt

Bochum

Bergstr. 26

44791 Bochum

ewig@augusta-bochum.de
Leitlinien nehmen aktuell einen wichtigen Stellenwert in der Etablierung medizinischer Standards ein. Ihnen kommt die ganze Autorität einer mit wissenschaftlichem Anspruch auftretenden Schulmedizin zu. Sie sind zum Kompass der medizinischen Praxis geworden, auch wenn der Grad der Umsetzung von Empfehlungen in Leitlinien nicht genau bekannt ist.

Die komplexe Problematik von Leitlinien, des Umfangs ihrer Berechtigung, ihrer Methodik, ihrer Qualität und ihrer Implementierung soll hier nicht reflektiert werden. An dieser Stelle soll lediglich ein Problem behandelt werden: die Handhabung von Interessenskonflikten der Autoren von Leitlinien. Hier zeichnet sich ein Konfliktfeld ab, das dringend einer umfassenden Diskussion auch in unserer Fachgesellschaft bedarf.

Welche Interessenskonflikte sind üblicherweise gemeint? Zuerst handelt es sich um die Konflikte, die aus der Tatsache entstehen, dass Autoren von Leitlinien in ihrer großen Mehrzahl Kontakte zur "Industrie“ (gemeint sind u.a. Hersteller von Pharmaka, von Medizinprodukten etc.) unterhalten. Als Wissenschaftler arbeiten sie mit Firmen zusammen, die bestimmte medizinische Produkte entwickeln und vermarkten, somit auf den Ebenen der Forschung ebenso wie auf der des Marketings. Diese Zusammenarbeit wird finanziell entgolten, in einem mittlerweile kodifizierten rechtlichen Rahmen, der zu einer aktuell hinreichenden politischen Akzeptanz und Rechtssicherheit geführt hat. Mögliche Interessenskonflikte in der Entwicklung von Leitlinien angesichts des Spannungsfelds von Industriekontakten einerseits und notwendiger Industrieneutralität andererseits wurden bis vor kurzem durch Offenlegung der Industriekontakte am Ende eines solchen Dokuments gehandhabt. Ansonsten wurde der mehr oder weniger strengen Methodik der Leitlinienerstellung zugetraut, eine hinreichende interessensfreie Sachlichkeit sicherzustellen.
Diese Verfahrensweise im Umgang mit Interessenskonflikten ist bereits Ergebnis einer Entwicklung, so wie die Methodik der Leitlinienerstellung weiterhin im Fluss ist. Die ersten Leitlinien waren methodisch wesentlich weniger streng und hatten aus heutiger Sicht mehr den Charakter einer Empfehlung von Experten, wobei wenig Sensibilität im Hinblick auf mögliche Interessenskonflikte bestand und daher mit dem Thema reichlich sorglos umgegangen wurde. So wurden noch vor rund zehn Jahren Leitlinien unter Beteiligung von Vertretern der Industrie formuliert. Heute ist die Sensibilität für mögliche Interessenskonflikte wesentlich höher.

Der Hintergrund der Verschärfung von Ansprüchen an die Interessensneutralität ist vielfältig und nicht ganz durchsichtig. Er hat sicherlich zu tun mit einer generellen Zunahme der moralischen Ansprüche an Personen in öffentlich relevanten Funktionen. Zwei weitere, ganz unterschiedliche Faktoren scheinen zudem eine Rolle zu spielen: zum einen die zunehmende Einflussnahme staatlicher Instanzen auf medizinische Prozesse, zum anderen der zunehmende Druck auf die Industrie, ihre Produkte zu refinanzieren und hinreichend profitabel zu machen. Der Einfluss außerklinischer Instanzen befördert eine eigentümliche Neigung, die Medizin rein theoretisch zu betrachten und die klinisch-wissenschaftlichen Akteure in einen vorgeblich interessefreien Raum zu projizieren. Das erforderliche Ausmaß der Investitionen in die Neuentwicklung und die damit verbundenen hohen Risiken führen dazu, den Raum für eine hinreichend selbstkritische Wahrnehmung des Stellenwertes eines Produktes immer weiter einzuschränken und führen zu früher so nicht gekannten fordernden, zuweilen auch aggressiven Werbestrategien der Industrie. In der Konsequenz stehen die Autoren von Leitlinien heute unter zunehmendem Rechtfertigungszwang. 
Dies geht in den USA so weit, dass die Autoren sich verpflichten müssen, für die gesamte Zeit der Leitlinienerstellung auf jegliche Einnahmen aus der Zusammenarbeit mit der Industrie zu verzichten, sofern sie mit Marketing in Zusammenhang gebracht werden kann. Dabei wird nicht mehr zwischen Einnahmen auf privaten, institutionellen oder wissenschaftlichen Konten unterschieden.

Zweifelsfrei kann man für diese radikale Forderung auf dem Hintergrund der erwähnten Refinanzierungszwänge und Marketingstrategien der Industrie gute Gründe anführen. Gegenargumente beziehen sich auch weniger darauf, Autoren von Leitlinien für generell interesseneutral zu erklären. Es gilt vielmehr auf die Fragwürdigkeit einer Forderung hinzuweisen, die außerhalb der ansonsten geltenden gesellschaftlichen und ökonomischen Plausibilitäten operiert. Während das Gewinninteresse als leitendes Motiv des Wirtschaftens unangefochten akzeptiert ist und Wissenschaftler entsprechend ausdrücklich zur Akquise von Drittmitteln aus der Industrie aufgefordert sind, werden ebendiese als Autoren von Leitlinien jetzt in die Pflicht genommen, die Kontakte zur Industrie zumindest temporär aufzugeben. Dabei ist es nicht besonders überzeugend, die Zusammenarbeit mit der Industrie in Forschung und Marketing streng zu trennen und Ersteres zuzulassen und Letzteres zu bannen, wenn man sich bewusst macht, dass kaum eine große Studie zustande kommt, bei der das wirtschaftliche Interesse nicht bis in die Fragestellung und das Design der Studie durchschlägt.

Die fehlende Plausibilität dieser Forderung ergibt sich zudem aus der Tatsache, dass die Distanz zur Industrie nur temporär gelten soll; inwieweit aber eine Zusammenarbeit mit der Industrie in dem Zeitraum vor der Leitlinienerstellung noch die Interessen beeinflusst und inwieweit nach der Leitlinienerstellung ein ehemals „Interessefreier“ nicht sogar seinen Marktwert für die Industrie sogar noch gesteigert finden kann, bleibt unreflektiert. Ein weiteres gewichtiges Bedenken gegen diese Forderung ergibt sich, wenn man ihre potenziellen Auswirkungen auf die Kollegialität der wissenschaftlichen Gemeinschaft ermisst. Schon jetzt zeichnet sich ab, dass diejenigen, die sich dieser Forderung nicht unterwerfen wollen, argwöhnisch auf die Kollegen schauen, die ihr zustimmen, und darin mitunter lediglich eine Art Vorteilsnahme von Karrierechancen sehen. Nicht auszudenken, wie es um die Glaubwürdigkeit von Leitlinien und ihrer Autoren, ja der gesamten wissenschaftlichen Gemeinschaft stünde, wenn scheinbares oder tatsächliches Abweichen von dieser Selbstverpflichtung öffentlich würde, und die mutmaßlich „Interessefreien" plötzlich als Scheinheilige dastünden.

Interessenskonflikte entstehen allerdings nicht nur auf der Ebene der Autoren, sondern auch auf einer Ebene, die bisher noch gar nicht diskutiert worden ist, nämlich auf der Ebene der Fachgesellschaften, die diese Leitlinien erstellen und vertreten. Der Anspruch einer Fachgesellschaft auf die Herausgabe einer Leitlinie ist jedoch eindeutig ein interessegeleitetes Vorgehen zugunsten der entsprechenden Berufsgruppe. Durch die Erstellung entsprechender Leitlinien werden „claims“ abgesteckt, um Ansprüche der Zuständigkeit für bestimmte Erkrankungen zu definieren. Unverkennbar stehen dabei die Empfehlungen unter dem Einfluss der Berufsgruppe, die die entsprechende Versorgung mit ihren Vorstellungen und Möglichkeiten abbilden möchte. Neuerdings finden sich sogar Beispiele, dass Leitlinien aus unterschiedlichen Gesellschaften gegeneinander konkurrieren und um Akzeptanz ihrer jeweiligen Methodik ringen, etwa auch hinsichtlich der Handhabung von möglichen Interessenskonflikten. Solche
Rivalität erzeugt jedoch neue, bisher nicht in das Augenmerk geratene Interessenskonflikte.

Desgleichen bringen auch Patientenorganisationen Interessen eigener Art ein, die klar benannt werden müssen.

Kurzum: das entscheidende Argument gegenüber einem radikalen Ansatz in der Handhabung möglicher Interessenskonflikte ist, dass dieser eben nur scheinbar radikal ist, vielmehr im Ergebnis in einem gewinnorientierten System der medizinischen Forschung nur neue Paradoxien und Gefahren erzeugen kann, je radikaler er sich gibt. Interessefreie Leitlinien sind somit definitiv nicht zu haben. Dies soll natürlich nicht heißen, dass alle Wissenschaftler konstitutiv interessegeleitet reden und handeln, oder sogar, wie von interessierter Seite sofort unterstellt würde, korrupt. Vielmehr soll die Komplexität der Aufgabe deutlich werden, die sich jedem stellt, der sich innerhalb eines gewinnorientierten Systems bewegen muss.

Aus unserer Sicht kann es im Kern keinen anderen Weg als den des Appells an das Gewissen der Autoren geben. Die Autoren sollten die Selbstkritik aufbringen, den Gehalt von Empfehlungen möglichst interessefrei zu halten. Die wissenschaftliche Gemeinschaft sollte dabei den Glauben an sich selbst, an das Gewissen jedes ihrer Mitglieder und der Gemeinschaft, aufrechterhalten. Nur dieser Glaube garantiert noch die Autonomie der medizinischen Willensbildung. Wenn wir an die Substanz eines solchen Gewissens nicht mehr glauben, sollten wir erst recht nicht auf die Kraft formaler Vorgaben setzen. Denn keine Formalität kann die innere Überzeugung ersetzen. Nur auf dem Hintergrund des Glaubens an das Gewissen der Akteure besteht im Falle eines Vergehens überhaupt Skandalfähigkeit, d. h. das Potenzial zur Selbstreinigung.

Es bedarf allerdings durchaus zusätzlicher formaler Regelungen, die den Umgang mit möglichen Interessenskonflikten pragmatisch, aber auch verbindlich regeln. An dieser Stelle möchten wir einige Vorschläge im Zusammenhang machen, die Anerkanntes und Neues einschließen.

1. Interessenskonflikte sollten transparent gehandhabt werden. Das heißt nicht nur, dass sie vollständig benannt werden, sondern auch klare Vorgaben zu formulieren, wo und wie genau sie benannt werden.

Interessenskonflikte einer Leitlinie ergeben sich aus den Fachgesellschaften, die sie vertreten, ebenso wie aus den Autoren, die sie erstellen. Somit sollte in jeder Leitlinie klar formuliert sein, welche Interessen die Fachgesellschaft mit der Leitlinie verfolgt. Sollten andere Fachgesellschaften dieselben oder ähnliche Themen beanspruchen, ist anzugeben, ob und in welcher Form eine Zusammenarbeit erfolgt oder eben nicht erfolgt ist, und im negativen Fall mögliche Gründe dafür aufgeführt sein.

Interessenskonflikte sollten nicht am Ende einer Leitlinie im Kleingedruckten, sondern am Anfang einer solchen im Text stehen.

Interessenskonflikte sollten so genau wie möglich genannt werden. Es genügt nicht, die Namen der Firmen aufzuzählen, sodass sich mit einer hohen Anzahl an Firmen sozusagen eine Neutralität eigener Art ergibt, Letztlich müssen Interessenskonflikte nicht nur benannt, sondern auch bewertet werden [1]. Aktuell sind weder Kriterien für eine Bewertung noch für die Auswahl der Bewertenden verfügbar. Vorgeschlagen wurden von der AWMF Bewertungen innerhalb der Leitliniengruppe sowie durch die Präsidien der Fachgesellschaften. Diese Vorschläge erscheinen jedoch wenig hilfreich, da sowohl Leitliniengruppen als auch Präsidien häufig selbst nicht frei 
von Interessenskonflikten sind, Letztere zuweilen gar Mitglieder der Leitliniengruppe. Überzeugendere Kriterien wären aus unserer Sicht 1) die exakte Angabe des Umfangs des Interessenskonflikts sowie 2) die objektive Graduierung des Interessenskonflikts anhand eines vorgegebenen Katalogs. Jeder Autor müsste demnach die Dimensionen der Drittmitteleinnahmen bzw.der persönlichen Honorierung in einem vordefinierten Zeitraum exakt angeben. Interessenskonflikte wären des Weiteren zu bewerten entsprechend der Intensität des entstandenen Interesses, also absteigend von Geschäftsanteilen/Eigentümerinteressen über Drittmittel/Beratertätigkeiten bis hin zu Vortrags-/Schulungstätigkeiten. Wo eine rote Linie zu ziehen wäre, jenseits dessen eine Mitgliedschaft in einer Leitliniengruppe ausgeschlossen wäre, gälte es zu diskutieren. Ganz sicher wäre sie zu ziehen im Falle von Geschäftsanteilen/ Eigentümerinteressen, im Falle der übrigen möglichen Interessenskonflikte am besten nach Umfang der Einnahmen.

2. Leitlinien sollten einer anerkannten Methodik folgen und entsprechende Verfahren der Leitlinienerstellung durchlaufen haben.

Selbstverständlich darf die Industrie keinerlei Einfluss auf die Finanzierung einer Leitlinie haben. Ebenso dürfen ihre Vertreter nicht Teil der Autorengruppe sein bzw. an Sitzungen der Autorengruppe teilnehmen.

Wenn Fachgruppen zu der Auffassung kommen, dass es keiner eigenen deutschsprachigen Leitlinie bedarf, weil eine qualitativ konkurrenzlose Leitlinie einer anderen international besetzten Fachgruppe vorliegt, können Sie Letztere natürlich in einer deutschsprachigen Übersetzung adaptieren. Jedoch kann die Übersetzung einer Leitlinie nur wörtlich ohne Änderungen akzeptiert werden. Jedwede Änderung des originären Textes, sei sie durch zwischenzeitlich neue Daten gerechtfertigt oder nicht, ist eine persönliche Meinung und nicht Teil der Leitlinie. Als Leitlinie kann nur ein Text akzeptiert werden, der über einen definierten formalen Prozess alle Aussagen auch eigenständig begründet hat.
3. Teil jeden Updates einer Leitlinie sollte eine Kritik der letzten Version sein. Leitend sollten dabei Fragen an die Qualität der eigenen Empfehlung sein. Dabei ist festzustellen, inwieweit eine andere Methodik zugrundegelegt wird. Empfehlungen zu Innovationen, die sich durch neuere Daten nicht haben aufrecht erhalten lassen, sollten ausdrücklich genannt werden.

An der Dringlichkeit einer Anpassung des Regelwerkes zur Erstellung von Leitlinien an neue Herausforderungen kann kein Zweifel bestehen. Diese Gedanken sind von der Hoffnung getragen, zu einer Meinungsbildung und möglichst baldigen klaren Positionierung auch innerhalb der „Deutschen Gesellschaft für Pneumologie und Beatmungsmedizin e.V.“ beitragen zu können.

\section{Interessenkonflikt \\ $\nabla$}

Alle Autoren sind Autoren von Leitlinien und Mitglieder der DGP sowie anderer medizinischer Fachgesellschaften. Alle Autoren haben z.B. Vortragshonorare von pharmazeutischen und medizintechnischen Unternehmen mit potenziellen Interessen bezüglich der Erstellung von Leitlinien erhalten.

Institute

1 Thoraxzentrum Ruhrgebiet, Kliniken für Pneumologie und Infektiologie, EVK Herne und Augusta-Kranken-Anstalt Bochum

2 Zentrum für Pneumologie, Diakoniekrankenhaus Rotenburg

${ }^{3}$ Klinik für Pneumologie, Medizinische Hochschule Hannover

${ }^{4}$ Medizinische Klinik 3 (Pneumologie, Allergologie, Schlafmedizin) am Klinikum Nürnberg

${ }^{5}$ Zentrum für Pneumologie, Psychosomatische Medizin und Psychotherapie, Universität Regensburg und Klinik Donaustauf

${ }^{6}$ Medizinische Klinik II - Klinik für Pneumologie, Intensiv- und Schlafmedizin Klinikum Oststadt-Heidehaus, Hannover

\section{Literatur}

1 Langer T, Conrad S, Fishman L et al. Interessenskonflikte bei Autoren medizinischer Leitlinien. Dtsch Ärzteblatt 2012; 48: 836-841 